\title{
Network based Mobility Management for Smart Homes through Proxy Mobile IP
}

\author{
Hyunju Munn, Kiho Lee, Ronnie D. Caytiles, and Byungjoo Park* \\ Department of Multimedia Engineering, Hannam University \\ 133 Ojeong-dong, Daeduk-gu, Daejeon, Korea \\ hyunju429@daum.net,kiholee@hnu.kr,rdcaytiles@gmail.com,bjpark@hnu.kr* \\ *Correspondent Author: Byungjoo Park* (bjpark@hnu.kr)
}

\begin{abstract}
One of the main requirements that need to be enhanced for smart home neworking is mobility. The capabilities of smart homes could be enormoust enhanced if equipped with mobility allowing the users an autonomous control over the home vevices and equipment. This paper presents a discussion of the concepts of proxy mobile IPv6 applied to smart home networks in order to spontaneously control the home devices and equipment while the home owner is mobile. An overview of the concepts of smart homes and proxy mobile IPv6 are also presented.
\end{abstract}

Keywords: proxy mobile IPv6, Smart Home, homeautomation

\section{Introduction}

The popularity of "Internet of Things" or Iot has brought the widely emerging smart home technology. The higher simplicity and affordability of home automation are achieved through the connectivity of smartphones, PDAs and tablets. These emerging developments in information technology have greatly affected on how people are dealing with their daily living chores.

The integration of information technology and services through home networking has made way to provide the houseblolds with a better quality of living and advantages that smart homes could offer [1]. The different home devices and equipment can now be remotely controlled through smartphones, PDAs and tablets.

This paper presents the correlation of the structure of Smart homes provided with mobility. The discussions on the overview of both the emerging technologies of smart homes and the standardized protocol for a network-based mobility management known as proxy mobile IPv6 are presented. The utilization of PMIPv6 for the implementation of the mobility of smart homes is also outlined.

The rest of this paper are organized as follows: Section 2 discusses the related technologies which provides the overview of the concepts of smart homes and how proxy mobile IPv6 works; the framework for the PMIPv6 based mobility of sensor nodes in a smart home is outlined in Section 3; and the concluding remarks in Section 4. 


\section{Related Technologies}

\subsection{Smart Home Systems}

Smart home systems allow the use of computer and information technology that enable occupants to remotely control and program home appliances and equipment. It is specially designed that ranges from simple remote control of lighting and windows to complex network of microcontroller based devices [2, 9]. It generally encompasses communications, entertainment, security, convenience, and information systems [9].

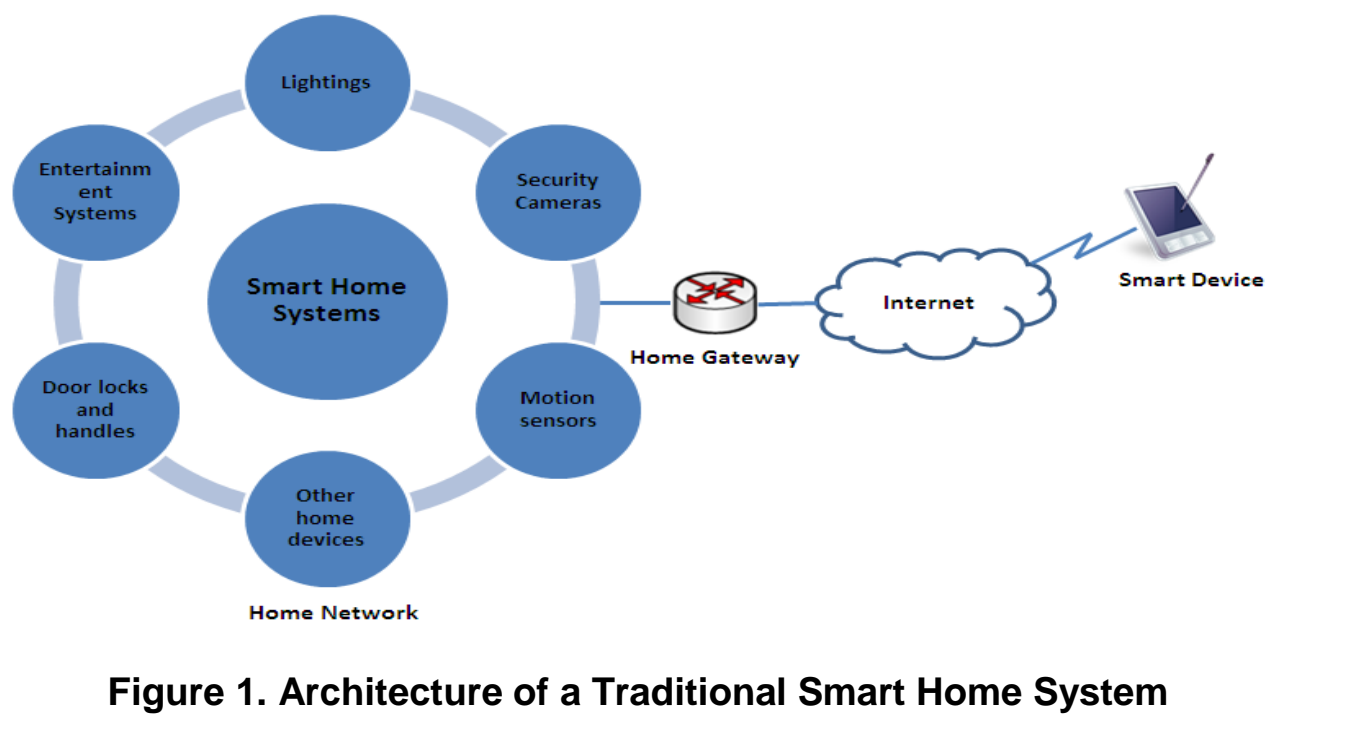

Traditional smart home systems are comprised of a home network, a home gateway, and the controller device or a mart device as shown in Figure 1. The home network consists of receivers, that is, the home devices and equipment that are wired or wirelessly connected together. The home gateway acts as the server that provides connection between these receivers and the Internet. All protocols for the operation of the receivers are defined in the home gateway wherein they are controlled by the users through their smart devices $[3,4]$.

\subsection{Proxy Mobile IPv6 Overview}

Mobile IPv6 refers to a protocol that allows mobile device users to move from one network to another while maintaining a permanent IP address. A mobile node (MN) in MIPv6 is addressed by two IP addresses, namely, a home address (HoA) and a care-of address (CoA) [5, 6]. HoA is a static IP address of a Mobile Node (MN) at its home subnet As the MN moves to a new subnet, it discovers the default router, perform address auto-configuration and duplicate address detection (DAD), and use its new address as CoA.

For the purpose of network-based mobility management, the Proxy Mobile IPv6 or PMIPv6 which is an IETF standardized protocol has been introduced. It enables the same functionality as of the mobile IP wherein the mobile node can change its point of attachment in an IP network without changing its IP address [7, 8]. Unlike in the conventional mobile IP, the network is responsible for the tracking of the movements and initiating the required mobility signaling for a mobile node (MN). 


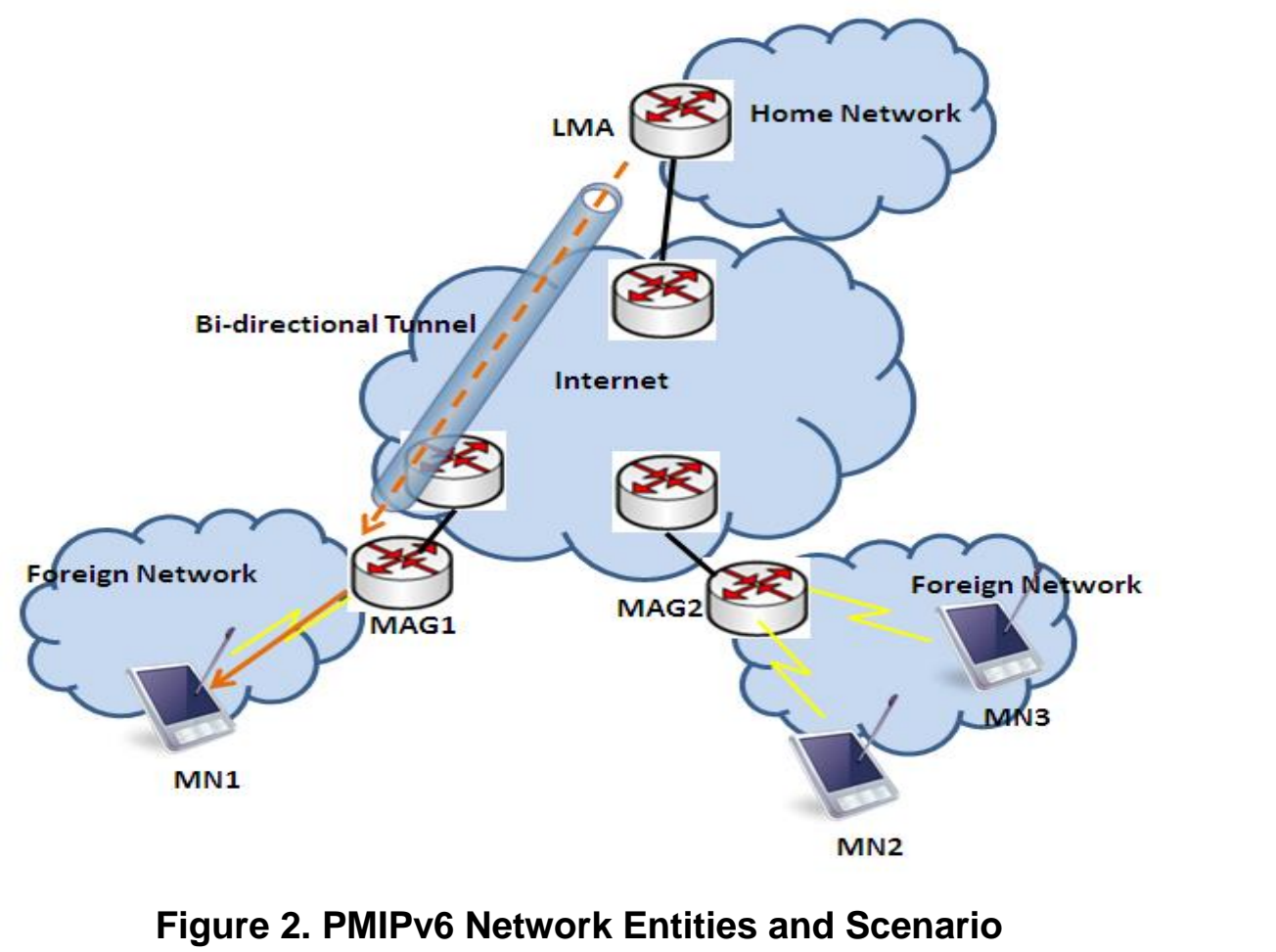

The functional network entities in the PMIP 6 network architecture are depicted in Figure 2 which includes the following [8]:

- The Mobile Access Gateway (MAG) performs the mobility-related signaling on behalf of the MyNs that ate attached to its access links.

- The Local Mobility Anchor (LMA) located within the home network maintains a collection of routes for each MN connected to the localized mobility domain (LMD).

The PMIP 6 performs the following protocol operations:

- A mobilehostenters a PMIP domain;

- A Mobile Access Gateway on that link checks host authorization;

- Almobile host obtains an IP address;

- A Mobile Access Gateway updates a Local Mobility Anchor about the current location of a host;

- Both MAG and LMA create a bi-directional tunnel;

- A Mobile Access Gateway sends a Router Advertise message to MN with Care-of-Address.

The mobile node attaches to an access link provided by the mobile access gateway (MAG) as it enters a PMIPv6 domain. Then the MAG on that link verifies the authorization of the MN to use the network-based mobility management service. Instead of the mobile node, the MAG then performs the mobility signaling by sending a proxy 
binding update (PBU) to the local mobility anchor (LMA) associating its own address with the mobile node's identity. On the other hand, the LMA sends a proxy binding acknowledgement (PBA) to the MAG that includes the prefix allocated to the Mobile Node. Both the MAG and LMA establishes a bidirectional tunnel.

The MAG sends Router Advertisement messages to the MN with the Care-of address in order for the $\mathrm{MN}$ to configure an address (stateless auto-configuration). The MN can alternatively use stateful address auto-configuration mechanisms. The IP packets that are to be transmitted by the correspondent node $(\mathrm{CN})$ intended to the mobile node $(\mathrm{MN})$ are intercepted by the LMA in the MN's home network and forward these IP packets to the MAG through the established bidirectional tunnel. The IP packets received by the MAG are de-capsulated and transmitted directly to the MN.

\section{Network based Mobility Management for Smart Homes}

The proxy mobile IPv6 can provide the smart home systems with networl based mobility management. The integration of PMIPv6 and wireless sensor netwoks have been the backbone for the design of the network based mobility management for smart homes. The traditional architecture of smart homes as indicated in Fig are l will be provided with mobility through the optimization of the concepts and principles of PMIPv6 as shown in Figure 3.

The integration of network based mobility management for smal homes is outlined in Figure 3 wherein the home user can control or issue controls for home devices and equipment attached to the home network even while he is moving around and is attached to foreign networks. The home user's smart device is associated with a care of address that identifies his current location that enables him to mainfin its connectivity as he enters a foreign network.

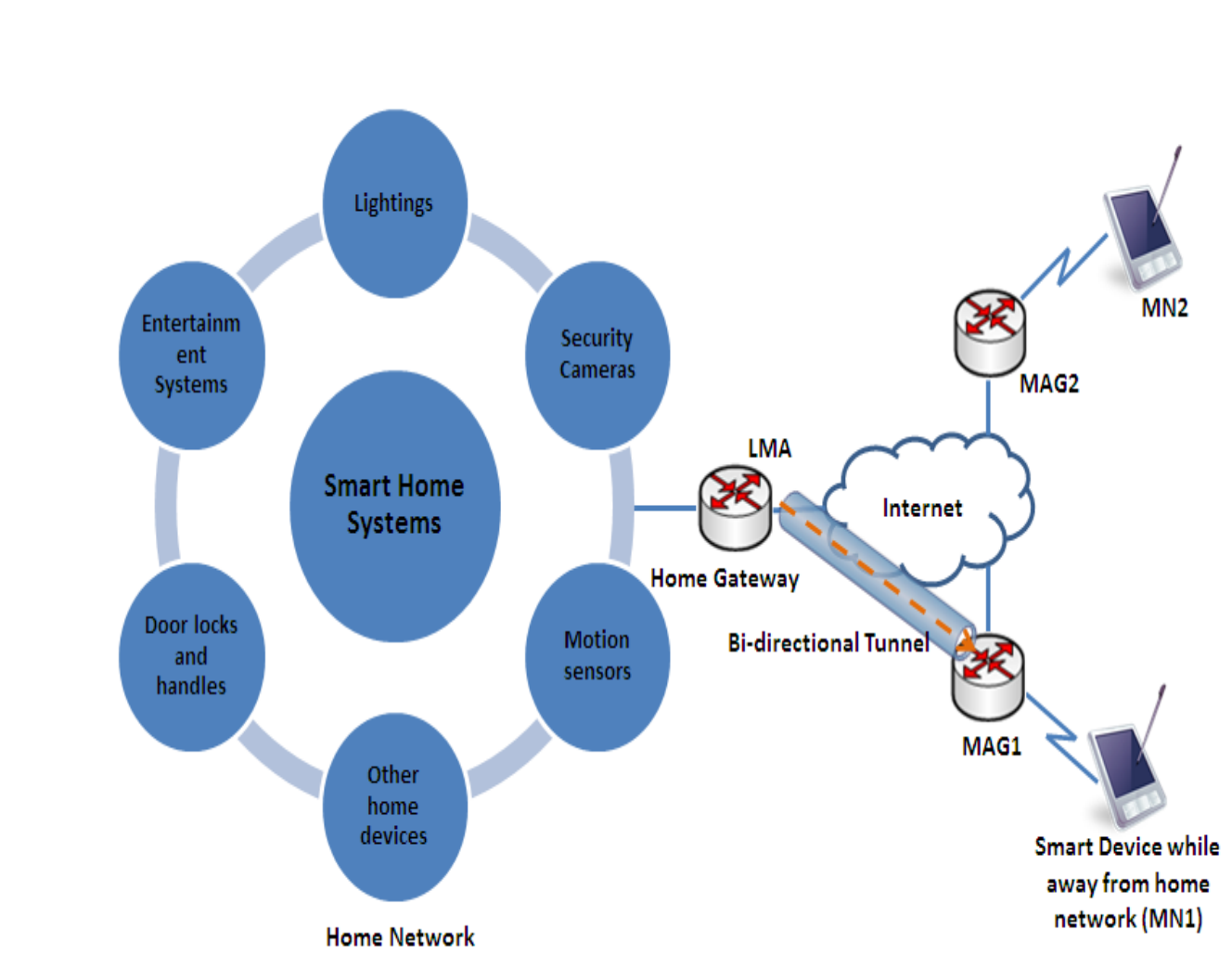

Figure 3. Network based Mobility Management for Smart Homes 
The home gateway that acts as the server or controller of the home devices and equipment attached to the home network will act as the local mobility anchor (LMA). The LMA will be responsible for routing the commands intended for each of the home devices and equipment directly to the mobile access gateway to which the smart device of the home user is currently attached.

The use of PMIPv6 message formats within the smart home system will be utilized for the communication among the home devices and equipment attached to the home network. Security protocols for IP will also be utilized for the authentication of the communication signals from home users to warrant the security and control of the home devices and equipment by the legitimate home owners.

Through the network based mobility management for smart homes, life for home owners may become easier and more convenient.

\section{Conclusion}

Optimized mobility is provided for smart home systems as the utilization of Proxy Mobile IPv6 enhances its communications and interconnection that lead to efficient, reliable, and emergent remote services a home networking has to of er. As the home owner is away, a seamless convergence of communication between the users and the home devices and equipment is provided by the proxy mobile IP based architectural design for smart homes. The remote home owner can have an autonomous controt over the home devices and equipment while he is mobile.

\section{References}

[1] Bierhoff, A. van Berlo, J. Abascal, B. Allen, A. Civit, K. Fellbaum, E. Kemppainen, N. Bitterman, D. Freitas and K. Kristiansson, "Smart home environment'Chttp://www.snapi.org.uk/cost219ter/ inclusive_future/ (14).pdf.

[2] R. J. Robles and T.-h. Kim, “Applications Sy stems and Methods in Smart Home Technology: A Review”, International Journal of Advanced Science and Technology, vol. 15, (2010) February, pp. 37-48.

[3] C. -L. Wu, C. -F. Liad and L. -C. Fu, "Service Oriented Smart-Home Architecture Based on OSGi and Mobile-Agent Technology", Systems, Man and Cybernetics, Part C: Applications and Reviews, IEEE Transactions, (2007), pp. 193-205.

[4] M. Edmonds, "How Smart Homes Work - Setting Up a Smart Home", (2011), Available at: http://home.how tuffworks.com/smart-home.htm, Accessed, (2014) October.

[5] http://en.wikipedia.org/wiki/Mrobile_IP.

[6] R. D. Caytiles, Y. E Gelogo and B. J. Park, "An Integrated Security Handover Scheme for Seamless Convergence Services over IP-based Mobile Networks", International Journal of Control and Automation, vol. 4, no. 4, (2010) December, pp. 55-62.

[7] http://en.wikipedia.org/wiki/Proxy_Mobile_IPv6.

[8] I. Soto, C. J. Bernardos, M. Calderón, T. Melia, "PMIPv6: A Network-Based Localized Mobility Management Solution", The Internet Protocol Journal, Volume 13, No.3, (2010) September, http://www.cisco.com/web/about/ac123/ac147/archived_issues/ipj_13-3/133_pmipv6.html.

[9] V. Redriksson, "What is a Smart Home or Building", (2011) April, Available at: http://searchciomidmarket.techtarget.com/definition/smart-home-or-building, Accessed: October 2014.

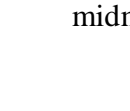

\section{Authors}

HyunJu Munn is a graduate student on the Master Course in Multimedia Engineering at Hannam University and one of the members of Mobile and Multimedia Communication Laboratory. Her research interests are Wireless Mobile Network (MIPv6, PMIPv6), IEEE 802.11f (Inter-Access Point Protocol), IEEE 802.16e (Mobile WiMAX), Wireless Sensor Network, Real-Time Multimedia Transmission Service. 
Kiho Lee is a graduate student on the Integrated Course in College of Engineering from Hannam University. His research interests include mobility management scheme, wireless network, mobile IPv6, multimedia system, Multimedia Architecture and sensor network.

Ronnie D. Caytiles is a graduate student on the Integrated Course in College of Engineering from Hannam University. His research interests include ubiquitous e healthcare, mobility management scheme, wireless network, mobile IPv6, multimedia system, Multimedia Healthcare, and sensor network.

Byungjoo Park received the B.S. degree in electronics engineering from Yonsei University, Seoul, Rep. of Korea in 2002, and the M.S. and Ph.D. degrees (Yirst-class honors) in Electrical and Computer Engineering from University of Florida, Gainesville, USA, in 2004 and 2007, respectively. From June 1, 2007 to February 28, 2009, he was a senior researcher with the IP Network Research Department, KT Network Technology Laboratory, Rep. of Korea. Since March 1, 2009, he has been a Professor in the Department of Multimedia Engineering at Hannam University, Daejeon, Korea. He is a life member of the IEEE, IEICE, IEEK, KICS, and KIISE. His primary research interests include theory and application of mobile comput $\mathrm{ng}$ including protocol design and performance analysis in next generation wireless/mobjle networks. He is an honor society member of Tau Beta Pi and Eta Kappa Nu

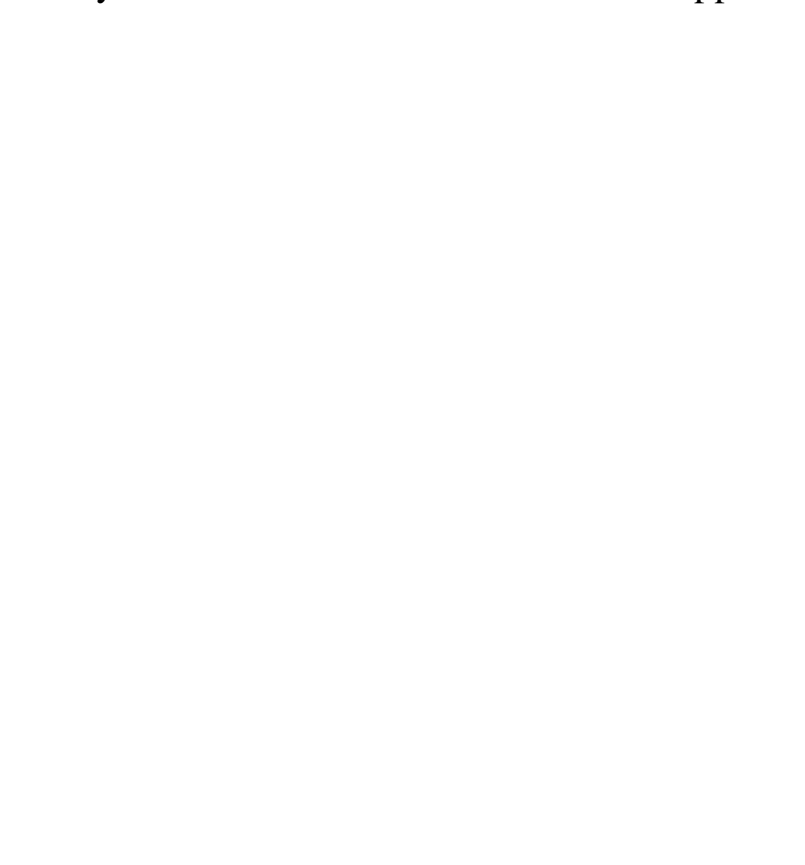

